Relations industrielles

Industrial Relations

\title{
Publications récentes
}

Recent Publications

Volume 15, numéro 1, janvier 1960

URI : https://id.erudit.org/iderudit/1022092ar

DOI : https://doi.org/10.7202/1022092ar

Aller au sommaire du numéro

Éditeur(s)

Département des relations industrielles de l’Université Laval

ISSN

0034-379X (imprimé)

1703-8138 (numérique)

Découvrir la revue

Citer ce document

(1960). Publications récentes. Relations industrielles / Industrial Relations, 15(1),

152-157. https://doi.org/10.7202/1022092ar

Tous droits réservés @ Département des relations industrielles de l’Université Laval, 1960
Ce document est protégé par la loi sur le droit d'auteur. L'utilisation des services d'Érudit (y compris la reproduction) est assujettie à sa politique d'utilisation que vous pouvez consulter en ligne.

https://apropos.erudit.org/fr/usagers/politique-dutilisation/ 
les, selon lui, devront se comporter dans ce domaine d'après des normes individuelles et non collectives, car les ingénieurs, savants et administrateurs dont l'industrie a besoin ne sont pas faits pour être moulés en série comme les travailleurs manuels.

Les deux communications suivantes présentent le point de vue de l'ouvrier et celui de lemployeur. Le rapport se termine par un exposé de Robert Cox sur certains aspects des relations industrielles sur le plan international et par une discussion entre experts.

G. C.

Frontiers of Industrial Relations. Edited by Robert D. Gray. Selected Papers published in observance of the Twentieth Anmiversary of the Industrial Relations Section, Cali- fornia Institute of Technology, $\mathrm{Pa}$ sadena, California, 1959, 458 pp.

La section des relations industrielles de l'institut de teohnologie de Californie a eu l'heureuse idée de célébrer son vingtième anniversaire en publiant cet ouvrage avec la collaboration de vingt-cinq auteurs dont les noms sont bien connus dans le monde des relations du travail. Les sujets abordés couvrent tous les aspects de $\infty$ domaine important et essaient de faire le point dans l'évolution actuelle.

L'ouvrage est présenté avec un espace pour prendre des notes. Ainsi, le lecteur peut-il facilement l'utiliser dans son travail. Il se vend au prix de $\$ 15.00$, lequel couvre aussi l'expédition de la seconde édition reliée qui paraîtra à l'été 1960.

\section{PUBLICATIONS RECENTES RECENT PUBLICATIONS}

\section{Généralités}

< Problèmes de la vieillesse et des vieillards », Chronique Sociale de France, 16, rue du Plat, Lyon, cahier 6 , ler actobre 1959, 67e année, pp. $394-447$.

— Propos sur le vieillissement $»$ par G. Gaillard, pp. 395-408.

— Psychologie du vieillissement chez l'homme », par Docteur J. Dublineau, pp. 409-430.

—La société et les vieillards \#, par Jean-Marie Arnion, pp. 431-447.

* La Formation Professionnelle des Adultes », par M. Bunel, Travail et Maîtrise, 4 rue Cambon, Paris ler, no 10 , novembre 1959 , p. 13.

«1. The Automobile Industry: A Case Study in Automation - 2. Automation and Leisure », by William A. Faunce, Automation and Society. 1959-60 Reprint Series, Labor and Industrial Relations Center, Michigan State University Publication, East Lansing, pp. 44-53, pp. 297-308.
- Canada in the Age of Steel s, by Stuart Armour, Canadian Labour, 100 Argyle Avenue, Ottawa 4, Vol. 4, No. 10, October, 1959, pp. 18-22.

Farm Labor Fact Book, U.S. Department of Labor, Washington, 240 pp.

\&L'orientation professionnelle en Bel. gique \$, Revue internationale du Travail, Genève, Suisse, vol. LXXX, no 6, décembre 1959, pp. 554-570.

¿The Maritime Industry », by Betty V. H. Schneider, Monthly Labor Review, United States Department of Labor. May 1959, Vol. 82, No. 5. Reprint No. 130, Institute of Industrial Relations, 201 California Hall, University of California, Berkeley 4. pp. 552557.

\section{Travail-Main-d'oeuvre}

Normes touchant l'emploi des ingénieurs professionnels, Corporation des ingénieurs professionnels de Québec, 1600 ouest, Ave Pine, Montréal. lère édition: Septembre 1959,36 pp. Edition française et anglaise. 
« Statut et rémunération du personnel médical des hôpitaux publics \$, par $F$. Montes, Formation, 26 rue de Montholon, Paris IXe, no 20, décembre 1959, 7 pp.

Age and Performance in Retail Trade: Two Case Studies of the Relations between Age and Selected Characteristics of Sales Personnel in Two Department Stores, Economics and Research Branch, Department of Labour, Ottawa, 16 pp.

c Immigration and Its Effect on Labor Force Characteristics \$, by Margaret S. Gordon, Monthly Labor Review, United States Department of Labor, May 1959, Vol. 82, No. 5. Reprint No. 129, Institute of Industrial Relations, University of California, 201 California Hall, Berkeley 4, California.

The Aging Worker in the Canadian Economy, Economics and Research Branch, Department of Labour, Ottawa, July 1959, 61 pp.

- Traitements des femmes dans l'enseignement supérieur \$, La Gazette du Travail, Ministère du Travail, Ottawa, nol. LIX, no 11, 30 novembre 1959, pp. 1216-1218.

- Campagne d'emploi pour les travailleurs âgés >, La Gazette du Travail, Ministère du Travail, Ottawa, vol. LIX, no 11,30 novembre 1959 , pp. 1220 1221.

c La formation professionnelle et technique en U.R.S.S. \$, par H. Zelenko, Revue internationale du Travail, Genève, Suisse, vol. LXXX, no 6 , décembre 1959, pp. 536-553.

\section{Direction du personnel}

« Manpower and Innovation: Some Pointers for Management », by Frederick Harbison, Personnel, NovemberDecember 1959, pp. 8-15.

\&hat Kind of Work Measurement Program? s, by R. C. Horne, Personnel, November-December 1959, pp. 25-34.

\& Personnel Records: Along the Road to Automation », by Paul Duke, Personnel, May-June 1959, pp. 31-40. c Must Market Pressure Wreck the Company's Salary Structure? ?, by Preston P. Le Breton, Personnel, JulyAugust 1959, pp. 34-45.

< Industrial Relations Budgets: Yardsticks for 1959 3. by Dale Yoder and Roberta J. Nelson, Personnel, JulyAugust 1959, pp. 16-27.

< Personnel Management in Theory and Practice », by Georges B. Strother, Personnel, May-June 1259, pp. 63-71.

< How to Appraise Executive Performance >, by Anch Patton, Harvard Business Review, January-February 1960, Vol. 38, No. 1, pp. 63-70.

< New Dimension in Measuring Morale $\gg$, by J. R. Glennon et al., Harvard Business Review, January-February 1960, Vol. 38, No. 1, pp. 106-7.

- Motivational Approach to Management Development 》, by Rensis Likert, Harvard Business Review, July-August 1959, Vol. 37, No. 4, pp. 75-82.

- What Can We Expect from Pensions? \$, by Daniel M. Holland, Harvard Business Review, July-August 1959, Vol. 37, No 4, pp. 125-140.

CReflections on Business >, by Paul F. Lazarsfeld, The American Journal of Sociology, July 1959, Vol. LXV, No. 1, pp. 1-31.

\& Organizational Goals and the Behavior of Informal Leaders », by Oscar Grusky, The American Journal of Sociology, July 1959, Vol. LXV, No. 1, pp. 59-68.

c Les procédures de licenciement: V. Royaume-Uni », Revue internationale du Travail, BIT, Genève, Vol. LXXX, no 4, octabre 1959, pp. 379-395.

\& La mobilita del lavoro e il ricambio del personale », por M. Corrado, Fattore Umano, Milano, via Senato 19. Anno IV, numero 10, ottobre 1959, pp. $680-685$.

< Ruolo e funzioni dello «staff 》 a livello direttivo 》, por M. Fertonani, Fattore Umano, Milano, via Senato 19. Anno IV, numero 10, ottobre 1959, pp. 662-675. 
\& Job Classification in the Steel Industry: The Experience of Two Local Unions », by Robert Dean Garton, IL Research, New York State School of Industrial and Labor Relations, Cornell University, Ithaca, New York, Volume V, Nos. 2 \& 3, Fall 1959, pp. 5-12.

« Merit Rating in Industry: A Survey of Current Practices and Problems 》, by Richard U. Miller, ILResearch, New York State School of Industrial and Labor Relations, Cornell University, Ithaca, New York, Volume V, Nos. 2 \& 3, Fall 1959, pp. 12-16.

\section{Organisation de l'entreprise}

\& Réflexions sur les conseils d'entreprise », par Albert Verschueren, Revue du Travail, Ministère du Travail, Belgique, octobre 1959, pp. 1305-1317.

«Authority Considered From An Operational Point of View 》, by Daniel J. Duffy, Journal of the Academy of Management, University of Illinois, Urbana, Ill. Volume 2, Number 3, December 1959, pp. 167-176.

\&A Critique of Business Games », by Billy E. Goetz, Journal of the Academy of Management, University of Illinois, Urbana, Ill. Volume 2, Number 3, December 1959, pp. 177-180.

«L'évolution du rôle du chef d'entreprise » par Jean-Marie Sedes, Jeune Patron, 19, Ave. Georges V, Paris 8e, no 130 , décembre 1959 , pp. 24-30.

«Quality Control System Gives Customers a Built-in Bonus », Plant Administration, 481 University Avenue, Toronto, Volume 19, No. 11, November, 1959, pp. 66-69.

\&Quick-Change Ideas And Top Methods Keep Costs Low », Plant Administration, 481 University Avenue, Toronto, Volume 19, No. 10, October, 1959, pp. 77-79.

- L'expérience de Bonpertuis d'association du personnel $»$, par Alexandre Dubois, Jeune Patron, 19, Avenue Georges $\mathrm{V}$, Paris 8e, no 129 , novembre 1959 , pp. $32-37$.
« La maîtrise et l'amélioration des. techniques de production $\gg$, par $\mathbf{M}$. Lavarde, Travail et Maîtrise, 4 rue Cambon, Paris ler, no 10, novembre 1959, pp. 8-9.

«Quelques études de simplification du travail », Concours pour les agents de maîtrise, par J. Benielli, Travail et Maîtrise, 4 rue Cambon, Paris ler, no 10 , novembre 1959 , p. 9.

\section{Questions économiques}

«Economics by Negotiation $\$$, by George $\mathrm{H}$. Hildebrand, The American Economic Review, Volume XLIX, May, 1959, Number 2. Reprint No. 82, Institute of Industrial Relations, University of California, Los Angeles 24, California, pp. 399-411.

«Labor's Participation in the European Productivity Program: A Study in Frustation », by William Gomberg, Political Science Quarterly, Vol. LXXIV, No. 2, June 1959. Reprint No. 127, Institute of Industrial Relations, University of California, 201 California Hall, Berkeley 4, California, pp. 240-255.

*Work Contacts and International Evaluations: The Case of a Mexican Border Village », by William H. Form and Julius Rivera, Social Forces, Vol. 37, No. 4, May, 1959. 1959-60 Reprint Series, Labor and Industrial Relations Center, Michigan State University Publication, East Lansing. pp. 334-339.

«Considérations sur le principe CA travail égal, salaire égal », par B. Frantzen-De Leye, Revue du Travail, Ministère du travail, Belgique. 60e année, septembre 1959, pp. 1140-1162.

«L'intéressement des travailleurs aux résultats de l'entreprise », Jeune Patron, 19, Avenue Georges V, Paris (8e). 13e année, no 128 , septembre-octobre 1959 , pp. 28-32.

* Une méthode d'intéressement aux résultats de l'entreprise », Jeune Patron, 19, Avenue Georges V, Paris (8e). 13e année, no 128 , septembre-octobre 1959, pp. 46-54. 
* L'intéressement des travailleurs à l'entreprise >, par H. Lesire-Ogrel, Formation, 26, rue de Montholon, $\mathrm{Pa}$ ris IX, 13e année, no 18, septembreoctobre 1959 , pp. 1-7.

« Pourquoi associer les travailleurs à la marche des entreprises? $\triangleright$, Jeune $\mathrm{Pa}$ tron, 19, Avenue Georges V, Paris (8e) 13 e année, no 128 , septembre-octobre 1959 , pp. $33-40$.

《Intéressement: Co-gestion? », par Hyacinthe Dubreuil, Jeune Patron, 19, Avenue Georges V, Paris (8e), 13e année, no 130 , décembre 1959 , pp. 4144.

«Top Cost-Cutting Ideas From Quebec 》, Plant Administration, 481 University Avenue, Toronto, Volume 19, No. 11, November, 1959, pp. 70-74.

«How to Control Inspection Costs », Plant Administration, 481 University Avenue, Toronto, Volume 19, No. 10, October, 1959, pp. 108-110.

«La productivité et l'emploi », La Gazette du Travail, Ministère du Travail, Ottawa, Vol. LIX, no 11, 30 novembre 1959, pp. 1189-1191.

«A Guide to Systematic Wage and Salary Administration \$, by Robert D. Gray, Industrial Relations Section, $\mathrm{Ca}$ lifornia Institute of Technology, Pasadena, California. Bulletin No. 29, May 1959, 38 pp.

«Wages Prices Profits Productivity », Report of the University of California, Western Assembly, Yosemite National Park, California, October 1-4, 1959. Institute of Industrial Relations, University of California, Berkeley. $10 \mathrm{pp}$.

\section{Relations industrielles}

«Reducing the Risks of Labor Arbitration s, by Paul Prasow, California Management Review, Vol. 1, No. 3, Spring, 1959. Reprint No. 83, Institute of Industrial Relations, University of California, Los Angeles 24, California, pp. 39-46.

«Négociations sur les salaires et politique des salaires en Suède: I $\gg, R e$ vue internationale du Travail, BIT, Genève, vol. LXXX, no 4, octobre 1959 , pp. 349-361.
《Quelques aspects du merit-rating ?, par P. Ghesquière, Bulletin social des industriels, 71 Ave. de Cortenberg, Bruxelles, no 261, novembre 1959, pp. 368-370.

«Evaluation des tâches et appréciation du mérite », par J. C. Dirick, Bulletin social des industriels, 71 Ave. de Cortenberg, Bruxelles, no 261, novembre 1959, pp. 371-376.

«Le merit-rating danis l'entreprise s, par C. Steenhoudt-Bosteels, Bulletin social des industriels, 71 Ave. de Cortenberg, Bruxelles, no 261, novembre 1959, pp. 377-378.

«Les conventions collectives en Belgique au cours de l'année 1959 (Note technique) », par C. Steenhoudt-Bosteels, Bulletin social des industriels, 71 Ave. de Cortenberg, Bruxelles, no 262, décembre 1959, pp. 391-400.

« Les conventions collectives à l'étranger », par J. Leblanc, Bulletin social des industriels, 71 Ave. de Cortenberg, Bruxelles, no 262, décembre 1959, pp. 401-409.

«Individual Rights in Collective Labor Relations », by Kurt L. Hanslowe, New York' State School of Industrial and Labor Relations, Cornell University, Ithaca, N. Y. Reprint Series No. 86, pp. 25-55.

« Government Intervention in Emergency Labor Disputes in Atomic Energy », by Robert C. Crawford, Labor Law Journal, CCC, Inc. Chicago 46, Ill. June 1959. Reprint Series, No. 85, New York State School of Industrial and Labor Relations, Cornell University, Ithaca, N. Y., pp. 414-434.

«Reducing the Risks of Labor Arbitration », by Paul Prasow, California Management Review, Vol. L, No. 3, Spring, 1959. Reprint No. 83, Institute of Industrial Relations University of California, Los Angeles 24, California, pp. 39-46.

« Major Trends in Labor Relations >, by Arthur M. Ross, Monthly Labor Review, United States Department of Labor, May 1959, Vol. 82, No. 5. Institute of Industrial Relations, Reprint No. 130, University of California, Berkeley 4, California, pp. 536-538. 
4 Association Bargaining \$, by Van Dusen Kennedy, Monthly Labor Review, United States Department of Labor, May 1959, Vol. 82, No. 5. Institute of Industrial Relations, Reprint No. 130, University of California, Berkeley 4, California, pp. 539-542.

< Preserving Management's Rights At the Bargaining Table $\gg$, by Jules $J$. Justin, Personnel, November-December 1959 , pp. 42-51.

\section{Sociologie-psychologie}

- Il est temps d'en finir avec la prétention mathématique en psychologie $\gg$, par L. Adam, Travail et Méthodes, 6 Ave. Pierre ler de Serbie, Paris, XVI, no 139 , novembre 1959 , pp. 53-55.

« Industrialization, Ideologies, and Social Structure $\gg$, by Reinhard Bendix, American Sociological Review, Vol. 24, No. 5, October, 1959. Institute of Industrial Relations, University of $\mathrm{Ca}$ lifornia, Berkeley 4, California, Reprint No. 132, pp. 613-623.

¿ Problems of Leadership 》, by J. A. McIntyre, The Canadian Personnel \& Industrial Relations Journal, 143 Yonge St., Toronto. Vol. 7, No. 1, January, 1960, pp. 15-19.

\& Management and Employee Motivation $\gg$, by Robert K. Burns, Public Personnel Review, April 1959. Industrial Relations Center, The University of Chicago, Reprint No. 91, pp. 122127.

\section{Syndicalisme}

Mémoire législatif présenté au gouvernement provincial du Québec, Fédération des travailleurs du Québec(CTC) en la ville de Québec, œ 11 novembre 1959, 57 pp. Edition française et anglaise.

Mémoire annuel de la CTCC au cabinet provincial, CTCC, 155 est Boulevard Charest, Québec, 4 novembre. 1959, 34 pp.

\& Organized Labor's Place in the Community Power Structure $\gg$, by William $\mathrm{H}$. Form, Industrial and Labor Relations Review, Vol. 12, No. 4, July 1959. 1959-60 Reprint Series, Labor and Industrial Relations Center, Michigan State University Publication, East Lansing, pp. 526-539.

Organized Labor and Political Action in Colorado: 1900-1960, by George E. Bardwell and Harry Seligson, College of Business Administration, University of Denver, Denver, Colorado, November, 1959, $67 \mathrm{pp}$.

\& L'évolution confessionnelle de la CTCC », par Jacques Cousineau, $R e$ lations, 8100 Boulevard St-Laurent, Montréal, no 228, décembre 1959, pp. 316-320.

Procès-verbal de la trente-hutitidme session du Congrès de la CTCC, CTCC, 155 est Boulevard Charest, Québec, $1959,318 \mathrm{pp}$.

Labour Organizations in Canada, Economics and Research Branch, Department of Labour, Ottawa, Forty-Eighth Edition, 1959, 92 pp.

«Three Images of Labour *, by William Dodge, Canadian Labour, 100 Argyle Ave, Ottawa, Vol. 4, No. 10, October, 1959, pp. 26-29.

«Structural Relationships Between Trade Unions and Labour Parties $\gg$, by Paul Malles, Canadian Labour, 100 Argyle Ave, Ottawa, Vol. 4, 10. Oo tober, 1959, pp. 30-37.

\& Union Strike Benefits: Steel Strike Helps Disclose - How Workers Sustain Selves », by Morris Maken, Industrial Bulletin, New York State Department of Labor, N.Y., November 1959, pp. 2-8.

\section{Législation du travail}

\&1. Secondary Boycott Loopholes 》, by Melvin J. Segal, Labor Law Journal, March, 1959. 1959-60 Reprint Series, Labor and Industrial Relations Center, Michigan State University Publication, East Lansing, pp. 175-202 and $\ll 2$. Differences Among Secondary Boycotts and the Taft-Hartley Act 》, Wayne Law Review, Vol. 5, No. 2, Spring 1959, pp. 195-208.

- Industrial Relations », Department of Labour, Ottawa, Annual Report for 
the fiscal year ended March 31, 1959, pp. 13-25.

$\rightarrow$ The Industrial Relations and Disputes Investigation Act 3, pp. 14-18.

- Canada Labour Relations Board , pp. 18-19.

$\longrightarrow$ Fair Wages Policy \$, pp. 20-22.

- Labour-management Co-operation Service 3, pp. 22-23.

$\rightarrow$ The Canada Fair Employment Practices Act \$, pp. 23-24.

$\rightarrow$ Female Employees Equal Pay Act \$, p. 24 .

- Annual Vacations Act \$, pp. 24-25.

«Relations industrielles », Ministère du travail, Ottawa, rapport annuel pour lannée financière terminée le 31 mars 1959, pp. 13-26.

- Loi sur les relations industrielles et les enquêtes visant les différends de travail s, pp. 14-18.

- Conseil canadien des relations ouvrières \$, pp. 18-20.

- Justes salaires dans les contrats du gouvernement 8, pp. 20-23.

$\rightarrow$ Service de collaboration ouvrière patronale s, pp. 23-24.

$\rightarrow$ Loi sur les justes méthodes d'emploi 3, p. 24.

- Loi sur l'égalité de salaire pour les femmes \$, pp. 24-25.

$\rightarrow$ Loi sur les vacances annuelles , pp. 25-26.

«Ĺ́gislation ouvrière \$, La Gazette du Travail, Ministère du Travail Ottawa, vol. LIX, no 11, 30 novembre 1959, pp. $1230-1238$.

$\rightarrow$ Lo Labor-Management Reporting and Disolosure Act s, pp. 1230-1233.

- Décision judiciaire d'intérêt ouvier 3, pp. 1233-1236.
- 18e réunion de l'Association canadienne des administrateurs de la législation ouvrière \$, pp. 12361237.

$\rightarrow$ Loi des fabriques au Royaume-Uni, $1959 \gg$, pp. 1237-1238.

¿Les procédures de licenciement 2, Reoue internationale du Travail, Go nève, Suisse, vol. LXXX, no 6, dó cembre 1959, pp. 571-587.

— VIII. Argetine », pp. 571-578.

- Japon 》, pp. 579-587.

\section{Sécurité sociale}

Statistiques du Travail et de la Sécurite Sociale, Ministère du Travail, 1, Place de Fontenoy, Paris VIle, octobre 1959, 74 pp.

Statistiques du Travail et de la Sécurité sociale, Ministère du Travail, 1, Place Fontenoy, Paris VIIe, novembre 1959, 91 pp.

\& Labor and Labor Relations on the West Coast: III $>$, Monthly Labor Review, United States Department of Labor, May 1959, Vol. 82 , No. 5 . Ro print No. 131, Institute of Industrial Relations, University of California, 201 California Hall, Berkeley 4, Cal. pp. 564-578.

\& 1. Unemplayment Disability Insurance in California \$, by Earl F. Cheit, pp. 564-571.

- 2. The Development of Health Insurance Plans », by Joseph W. Garbarino, pp. 572-578. 